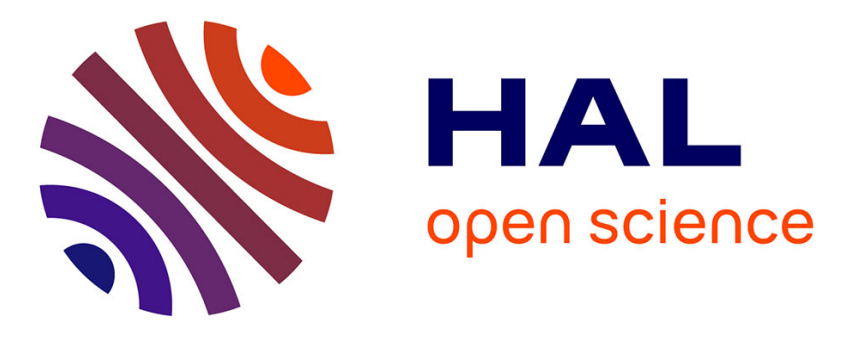

\title{
Des listes pour apprendre, résoudre, classer, archiver, explorer ou inventer \\ Christine Proust
}

\section{To cite this version:}

Christine Proust. Des listes pour apprendre, résoudre, classer, archiver, explorer ou inventer. Brooke Holmes; Klaus-Dietrich Fischer. The Frontiers of Ancient Science: Essays in Honor of Heinrich von Staden, De Gruyter, pp.493-514, 2014. hal-01139604

\section{HAL Id: hal-01139604 https://hal.science/hal-01139604}

Submitted on 6 Apr 2015

HAL is a multi-disciplinary open access archive for the deposit and dissemination of scientific research documents, whether they are published or not. The documents may come from teaching and research institutions in France or abroad, or from public or private research centers.
L'archive ouverte pluridisciplinaire HAL, est destinée au dépôt et à la diffusion de documents scientifiques de niveau recherche, publiés ou non, émanant des établissements d'enseignement et de recherche français ou étrangers, des laboratoires publics ou privés. 


\section{Textes mathématiques cunéiformes: des listes pour apprendre, résoudre, classer, archiver, explorer ou inventer}

\section{Christine Proust (CNRS \& Université Paris Diderot)}

Résumé - Les textes mathématiques de Mésopotamie sont généralement écrits sous forme de listes, comme le sont la plupart des textes savants provenant du Proche Orient ancien. Du fait même de leur structure, les listes mathématiques véhiculent des significations particulières qui méritent d'être étudiées en tant que telles. Dans cette contribution, je m’intéresserai principalement à des textes composés de longues listes d'énoncés de problèmes. Bien que présentant des ressemblances formelles, ces listes sont très diverses et reflètent des projets intellectuels différents. Certaines listes d'énoncés sont des "catalogues", qui semblent avoir pour but d'inventorier, classer et probablement archiver un matériel mathématique existant, largement utilisé dans l'enseignement. D'autres sont de très longues listes de plusieurs milliers d'énoncés couvrant des séries de plusieurs tablettes numérotées. Dans ces "séries" de structure hautement élaborée, les scribes ont produit du matériel mathématique entièrement nouveau. La démarche de ces scribes paraît être alors plutôt heuristique que classificatoire.

Note liminaire - Cette contribution résulte de recherches sur "l'art des listes" dans les mathématiques cunéiformes que j'ai pu développer à l'Institue for Advanced Studies en 2009 dans le cadre d'un membership financé par la fondation Neugebauer. Ces travaux seront publiés dans différents articles et ouvrages ${ }^{1}$. La présente contribution se limite à un aspect des listes mathématiques, leur statut pragmatique. Pour plus de détails sur d'autres aspects, je renvoie à ces publications. Ma réflexion s'est considérablement enrichie du contexte intellectuel de l'IAS, notamment au travers des discussions qui ont accompagné les deux exposés que j’ai pu donner pendant mon séjour. Le premier exposé, intitulé «Why do colophons matter? Old Babylonian mathematical tablets » était programmé dans le cadre des "Ancient Studies seminars» animé par Heinrich von Staden, que je remercie particulièrement. Le deuxième, intitulé "The art of list », est intervenu dans le cadre du séminaire intitulé «Historical Studies Lunchtime Colloquia » du département d’histoire de l'IAS. Le contenu de la présente contribution s'inspire largement de ces deux exposés et des échanges d'idées qu’ils ont suscités. Les outils plus strictement linguistiques que j’ai utilisés dans ce travail ont été forgés grâce à cinq ans de séminaires sur «histoire des sciences, histoire du texte » organisés à Paris par Jacques Virbel et Karine Chemla, envers qui ma dette intellectuelle est considérable. Le matériau textuel, ainsi que le recul nécessaire à une approche globale des listes, ont été acquis grâce à l'accueil de l'Institut for the Study of the Ancient Word et l'Unité Mixte de Recherche (CNRS) de New York University en 2010, et à ma résidence à l’Institut Méditerranéen d’Etudes Avancées de Marseille en 2010-2011. Enfin, je n'ai pu étudier directement les sources qui sont à la base de mon travail sur les listes que grâce à l'accès aux collections de tablettes cunéiformes qui m’a été généreusement offert par

\footnotetext{
${ }^{1}$ Proust en préparation ; Proust 2012.
} 
Béatrice André-Salvini pour le Louvre, et par Benjamin Foster et Ulla Kasten pour la Yale Babylonian Collection.

\section{L'art des listes}

L’organisation du savoir sous forme de listes est si générale en Mésopotamie qu'elle a été perçue, dans les débuts de l'assyriologie, comme une forme de pensée caractéristique du Proche Orient Ancien, comme un trait de civilisation. Cette approche unificatrice est encore très présente dans les études actuelles. Pourtant, elle me paraît insatisfaisante pour au moins deux raisons. D’une part la façon dont les listes ont été créées et utilisées a connu des variations considérables au cours de la longue histoire de l'écriture cunéiforme, selon les contextes, les époques, les genres de textes. Des listes d'apparences très similaires peuvent résulter de démarches tout à fait différentes, et c'est ce point précis que je voudrais mettre en évidence ici dans le cas des listes mathématiques. D’autre part les listes ne sont pas spécifiques au Proche Orient Ancien. Elles sont omniprésentes dans toutes les traditions écrites, et, dans une certaine mesure dans les traditions orales. Aujourd'hui, les listes ont envahi notre quotidien, par exemple avec les bases de données et les documents stockés dans nos ordinateurs.

C’est sans doute Jack Goody qui, le premier, a souligné l'importance des listes dans les écrits anciens. Pour lui, la liste est même une sorte d'archétype de l'écrit à ses débuts ${ }^{2}$. Ce n'est pourtant que récemment que cette forme de texte banale et apparemment anodine est devenue un objet d'étude en tant que tel. Les études récentes des listes, et plus généralement des énumérations, portent sur des corpus de tous genres et de toutes époques ${ }^{3}$. De telles études me paraissent essentielles pour progresser dans la compréhension des textes anciens.

Pour étudier les structures de liste dans la documentation cunéiforme, il est intéressant d'accorder une attention particulière aux textes mathématiques. En effet, la logique interne aux mathématiques permet de saisir des mécanismes de création des listes qui ne sont pas toujours clairs dans d'autres genres de textes, notamment dans les textes de divination. De plus, les mathématiques cunéiformes nous offrent ce que les savants de Mésopotamie ont produit de plus élaboré en matière de liste. Dans certains textes mathématiques, on voit cette « science » textuelle se déployer avec une virtuosité spectaculaire, comme on le verra dans ce qui suit. Mais, curieusement, cet aspect n’a pas attiré l'attention des historiens des mathématiques. Ces derniers se sont concentrés sur les résultats mathématiques, par exemple sur les méthodes de résolution des problèmes quadratiques, mais se sont peu intéressés aux structures textuelles. L'unité de texte examinée par les chercheurs modernes est principalement le problème, considéré de façon isolée, indépendamment de l'ensemble des problèmes qu'un scribe a choisi de réunir et de noter sur une même tablette, dans un ordre précis. A de rares exceptions près, il n’a guère été tenu compte du fait que les problèmes constituaient des listes. Or certaines informations capitales résident précisément dans la

\footnotetext{
${ }^{2}$ Goody 1977.

${ }^{3}$ Luc, Mojahid, Pery-Woodley et al. 2000; Virbel 1997 ; Virbel à paraître. Voir aussi Sève 2010 et sa bibliographie.
} 
structure de ces listes. Une analyse des structures de liste est particulièrement utile pour les textes mathématiques qui ne contiennent pas de procédure de résolution (on verra des exemples plus loin). Dans ces cas, le contenu mathématique ne réside pas dans la résolution des problèmes, puisque celle-ci est absente, mais dans la façon dont les énoncés sont construits et agencés. Or cette construction ne se décèle pas à l'échelle d'un seul énoncé, mais à l'échelle de tout un ensemble d'énoncés, ensemble qui résulte parfois d'un mécanisme générateur unique.

Mais qu'est-ce qu'une liste? Les listes ou, plus généralement, les structures énumératives, sont si multiformes qu'elles défient les tentatives de définition. La diversité des formes et des usages des listes est extrême. Au-delà des problèmes de définition, qui ne sont peut-être pas essentiels, ce sont des questions plus pragmatiques qui me semblent pouvoir apporter une aide dans la compréhension des textes anciens : quel type de message un scribe voulait-il exprimer ou transmettre lorsqu'il notait une liste sur une tablette d'argile ? Il est bien sûr difficile de répondre à une telle question puisque les scribes n'ont guère laissé de traces explicites de leurs intentions. Cependant, je voudrais montrer, par les quelques analyses qui suivent, que le but n'était pas toujours, comme on le suppose souvent, de transmettre un savoir, c'est-à-dire d'exposer des connaissances ou de donner des instructions pour la résolution d'un problème. Les scribes pouvaient poursuivre des buts autres, comme par exemple l'archivage, ou certaines formes de réflexion théorique, ou encore des tentatives d'exploration de domaines qui leurs étaient inconnus.

\section{Les mathématiques cunéiformes: quelques repères et quelques questions}

Les textes mathématiques écrits sur argile qui ont été découverts en Mésopotamie et dans les régions voisines représentent plus de 2000 tablettes ou fragments. L'immense majorité d'entre eux date de la période paléo-babylonienne $(=\mathrm{pB}$, début du deuxième millénaire avant notre ère), dont il sera essentiellement question dans ce qui suit. Une partie considérable de ce corpus est constitué de «tablettes scolaires », c'est-à-dire de tablettes écrites par des écoliers au cours de leur formation élémentaire. Les autres documents mathématiques peuvent être qualifiés de «savants » par opposition aux écrits d'écoliers. Ce sont des textes plus ou moins érudits, sans doute écrits par des étudiants avancés ou des maîtres d'écoles de scribes. La fonction de certains de ces textes est reconnaissable par les caractéristiques matérielles des tablettes d'argile sur lesquels ils sont écrits. Cette typologie est bien connue pour les textes scolaires élémentaires, mais moins claire pour les textes d'enseignement avancés ou d'érudition. Dans le domaine des mathématiques et de la littérature, on peut néanmoins noter une certaine régularité. Il semblerait que, dans les niveaux avancés de l'enseignement, maîtres et étudiants utilisaient des tablettes écrites sur une seule colonne, dites de «type $\mathrm{S}$ ». Des tablettes multi-colonnes, dites de «types $\mathrm{M}$ », étaient pour des usages qui semblent plus variés, par exemple des examens ou des travaux de pure érudition. Cette typologie n'est en fait véritablement établie que pour les textes littéraires de 
Nippur $^{4}$ (voir S. Tinney), mais elle peut apporter des indices, à recouper avec d'autres, pour identifier les auteurs de textes de provenance autre ou inconnue.

Du fait que les mathématiques d'époque pB sont essentiellement issues des activités des écoles de scribes, ces textes sont généralement interprétés par les historiens comme relevant d'une transmission de maître à élève. Pourtant, un tel modèle de communication, qui se limite à la transmission de haut en bas, ne permet pas de comprendre des phénomènes tels que la circulation des idées, la standardisation du cursus de formation, les voies originales de l'invention mathématique. Dans l'étude des listes mathématiques cunéiformes qui suit, je voudrais montrer au contraire la diversité des modèles de communication.

Les textes mathématiques savants se partagent en plusieurs catégories selon leur contenu, leur structure, le type de tablettes sur lesquelles ils sont écrits, leur langue, leur provenance, leur datation, etc. ${ }^{5}$. Je ne vais pas entrer ici dans le détail de ces classifications ${ }^{6}$. Je m'intéresserai seulement à trois catégories qui, d’une certaine façon, ont été reconnues comme telles par leurs auteurs dans la mesure où ces catégories portent des noms sumériens ou akkadiens. La première de ces catégories comprend les textes de procédure (kibsum en akkadien), qui sont des listes d'énoncés de problèmes suivis d'une procédure de résolution détaillée. La deuxième catégorie est formée des catalogues, qui sont des listes d’énoncés de problèmes mais ne contiennent aucune indication pour la résolution; ils se terminent généralement par une brève notice (colophon) indiquant le nombre d'énoncés $(\mathrm{N}$ im-šu $=\mathrm{N}$ sections) et, souvent, le sujet de ces énoncés (champs, ou briques, ou canaux, etc.). La dernière catégorie est, comme la précédente, composée de listes d'énoncés sans procédure de résolution. Cependant, contrairement à celles des catalogues, ces listes se déploient sur plusieurs tablettes (une dizaine ou plus), chacune portant un numéro, comme les pages d'un livre, et sont appelées « séries » en assyriologie. En examinant chacune de ces trois catégories de textes, j'essaierai de montrer la diversité des buts poursuivis par les scribes et des publics auxquels ils s'adressaient.

\section{Des listes pour résoudre des problèmes : les textes de procédure}

Les textes mathématiques les plus nombreux, ou en tous cas les plus étudiés, sont des listes de problèmes résolus. Le plus souvent, les énoncés sont rédigés à la $1^{\text {ère }}$ personne, et les procédures de résolution sont à la $2^{\mathrm{e}}$ personne. La procédure se présente comme une liste de consignes, ouverte par une formule figée : «toi, pour le savoir », et fermée par une autre formule figée : «telle est la façon d’opérer ». Les problèmes d'un texte de procédure portent

\footnotetext{
${ }^{4}$ Tinney 1999 ; Delnero 2010.

${ }^{5}$ Note sur les langues: les deux langues utilisées dans les textes mathématiques (et d'une façon générale dans les textes cunéiformes d'époque pB provenant de Mésopotamie), sont le sumérien et l'akkadien. Le sumérien est une langue de type agglutinant, de groupe linguistique inconnu, qui était parlée en Mésopotamie du sud au cours du $3^{\mathrm{e}}$ millénaire, et qui a probablement disparu des usages courants avant l'époque pB pour devenir une langue d'érudition enseignée dans les écoles de scribes. L'akkadien est une langue sémitique qui, à l'époque pB, était la langue maternelle d'une grande partie de la population de Mésopotamie.

${ }^{6}$ Pour un classement des sources mathématiques selon l'origine géographique, voir (Goetze 1945) et (Høyrup 2002, ch. 8 et 9). Pour une discussion sur les problèmes posés par la classification du matériel mathématique cunéiforme et une bibliographie associée, voir Proust 2012 et Proust en préparation.
} 
généralement sur une même thématique : surfaces de champs, creusement de canaux, volume de tas de briques, opérations commerciales, ou autre. Une liste de problèmes peut être obtenue par différents procédés. Parfois, les problèmes sont emboîtés, chacun reprenant le précédent en ajoutant un élément de complexité. Parfois, les données du problème varient, ce qui permet d'explorer tous les aspects d'une situation donnée, ou bien tous les cas possibles. Parfois, la situation imaginée (par exemple le creusement d'un canal) est la même dans tous les problèmes de la liste (par exemple, mêmes dimensions du canal, même nombre d'ouvriers, mêmes salaires...), et les différents énoncés sont obtenus par permutation circulaire des paramètres.

Un exemple de ce dernier procédé est fourni par la tablette YBC 4663, d’époque pB, d’origine inconnue et conservée à Yale. Elle contient 8 problèmes concernant le creusement d'une tranchée. Les énoncés sont écrits en sumérien (caractères droits dans la traduction cidessous), et la procédure est écrite en akkadien (mots en italiques). La traduction des deux premiers problèmes est la suivante ${ }^{7}$ :

$\# 1$

1. Une tranchée. Sa longueur est 5 ninda, sa largeur est 1 1/2 ninda, sa profondeur est $1 / 2$ ninda. Le volume assigné (à chaque ouvrier) est 10 gin $_{2}$. Le salaire (d'un ouvrier) est 6 še.

2. Que sont la base, le volume, le nombre d'ouvriers et l'argent (salaire total) ? Toi, pour le savoir :

3. La longueur et la largeur croise, cela te donnera 7.30.

4. $\quad 7.30$ à la profondeur élève, cela te donnera 45 .

5. L'inverse du volume assigné dénoue, cela te donnera 6. A 45 élève, cela te donnera 4.30

6. 4.30 au salaire élève cela te donnera 9. Telle est la façon d'opérer.

\#2

7. $9 \operatorname{gin}_{2}$ l'argent (total) pour la tranchée. 1 1/2 ninda sa largeur, 1/2 ninda sa profondeur. 10 gin $_{2}$ le volume assigné. 6 še (d'argent) le salaire (d'un ouvrier).

8. Sa longueur combien ? Toi, pour le savoir : la largeur et la profondeur croise.

9. 9 te donnera. L’inverse du volume assigné dénoue,

10. à 9 élève. 54 te donnera.

11. 54 au salaire élève. 1.48 te donnera.

12. L'inverse de 1.48, 33.20 te donnera. 33.20 à 9, l'argent (total), élève.

13. 5 te donnera. 5 ninda sa longueur. Telle est la procédure.

Le problème 2 dérive du problème 1 par une simple permutation circulaire des paramètres: certaines données deviennent des inconnues et vice-versa. Les six autres problèmes de la tablette résultent du même procédé. La procédure de résolution proprement dite est une liste de consignes encadrée par les deux formules qui ouvrent et ferment l'algorithme : «Toi, pour le savoir » et «Telle est la façon d’opérer ». Ces deux formules

\footnotetext{
${ }^{7}$ Traduction personnelle, basée sur Neugebauer et Sachs 1945: 70.
} 
soulignent la nature prescriptive de la liste d'instructions. On le voit, les textes de procédure sont des listes (de problèmes) renfermant d'autres listes (de consignes).

Les textes de procédure ont généralement pour but de montrer comment on résout un type de problème donné, représenté par une collection de cas particuliers. Dans le cas de la tablette YBC 4663, une telle démarche s'inscrit probablement dans un contexte d'enseignement. Le fait que la tablette ne comporte qu'une seule colonne (type S) donne à penser qu'elle a été écrite par ou pour un étudiant avancé, et donc confirme le caractère didactique du texte. Il convient toutefois de noter que les textes de procédure ne reflètent pas toujours aussi clairement des activités d'enseignement et, dans certains cas, peuvent s'inscrire dans des réseaux de relations entre érudits non liés strictement à l'enseignement ${ }^{8}$.

\section{Des listes pour classer et archiver : les catalogues}

Les catalogues sont des listes d'énoncés de problèmes, ne donnant aucune indication sur la façon de les résoudre. Les problèmes listés dans les catalogues se retrouvent généralement dans les textes de procédure, sous des formes similaires ou même absolument identiques comme on va le voir dans ce qui suit. Les auteurs des catalogues rassemblaient dans une tablette des énoncés de problèmes relatifs à un même sujet, comme le creusement des canaux, ou bien des surfaces de champs, ou bien des calculs de volume relatifs à des briques, etc.

\footnotetext{
${ }^{8}$ Voir à ce sujet (Bernard and Proust in progress).
} 


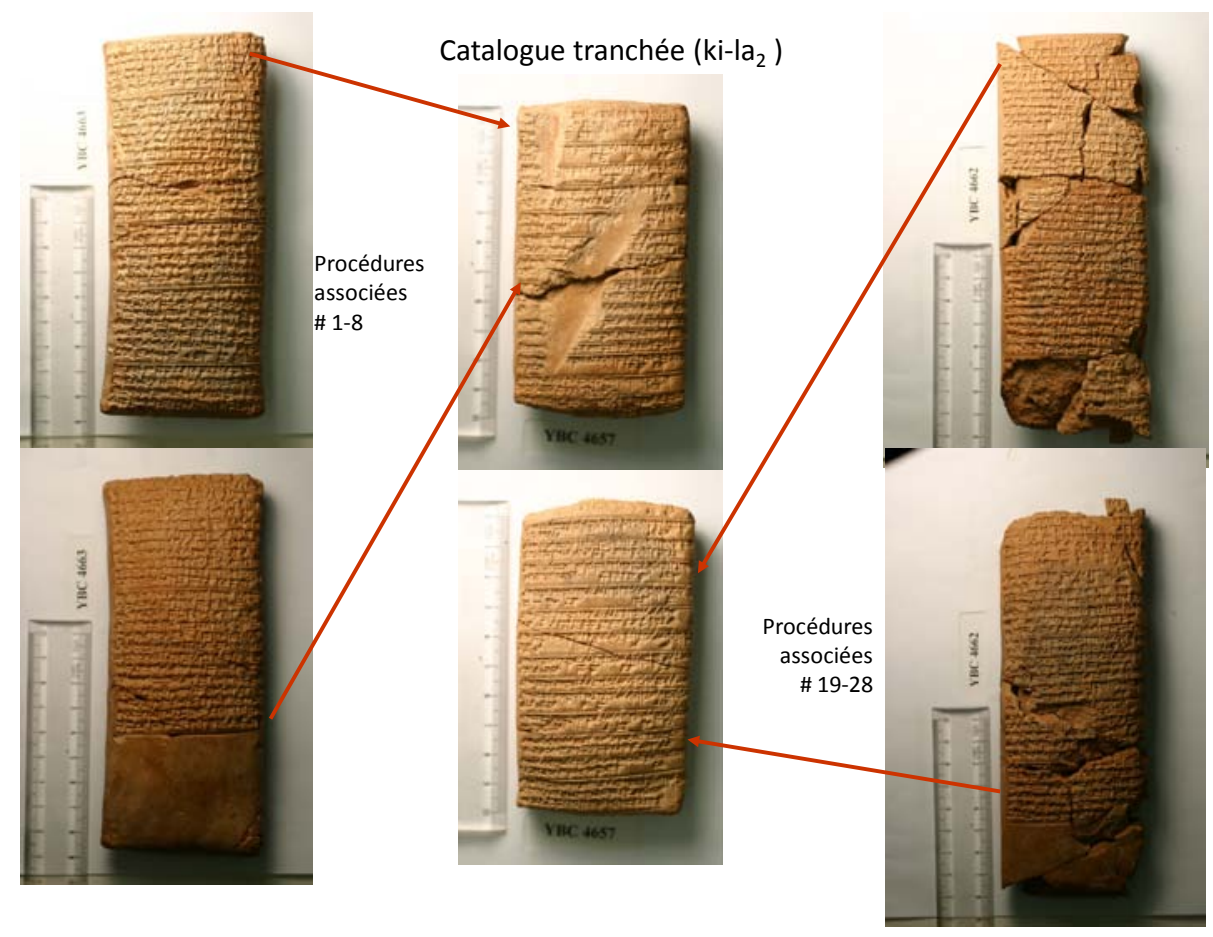

Figure 1 : Catalogue (au centre) et textes de procédure associés (à gauche et à droite)

Voici par exemple la traduction du début du catalogue YBC 4657 contenant 31 énoncés de problèmes concernant le creusement d’une tranchée :

\#1

Une tranchée. 5 ninda sa longueur, 1 1/2 ninda sa largeur, 1/2 ninda sa profondeur. 10 gin $_{2}$ le volume assigné (à chaque ouvrier). 6 še (d’argent) [le salaire d'un ouvrier].

La base, le volume, le nombre d'ouvriers et l'argent (des salaires) combien ? 7 $1 / 2$ la base, 45 le volume.

$4 \times 60+30$ [ouvriers], 9 gin $_{2}$ l'argent (des salaires).

\#2

L’argent (total) pour la tranchée est [9 gin2]. 1 1/2 ninda sa largeur, 1/2 ninda sa profondeur. 10 gin 2 la tâche assignée. 6 še (d’argent) le salaire d’un ouvrier. Sa longueur [combien] ? 5 ninda sa longueur.

\#3

L’argent (total) [pour la tranchée est 9 gin $_{2}$ ]. 5 ninda sa longueur, 1/2 ninda sa profondeur. 10 gin 2 la tâche assignée. 6 še (d'argent) le salaire d’un ouvrier.

Sa largeur combien ? 1 1/2 ninda la largeur.

\# 4

L'argent (total) pour la tranchée [est 9 gin $_{2}$ ]. 5 ninda sa longueur, 1 1/2 ninda sa largeur. 10 gin $_{2}$ la tâche assignée.

6 še (d'argent) le salaire (d'un ouvrier). Sa profondeur combien ? [1/2 ninda] sa profondeur. 
La tablette se termine par un colophon ainsi libellé : 31 sections $^{9}$ (im-šu) à propos de tranchées $\left(\mathrm{ki}^{-} \mathrm{la}_{2}\right)$. Le colophon précise donc le nombre de problèmes contenu dans la tablette et le thème des problèmes.

Si le texte est homogène du point de vue du thème des énoncés, qui tous concernent le creusement d'une tranchée, ils ne le sont pas d'un point de vue mathématique. On distingue assez nettement quatre groupes différents ${ }^{10}$. Le premier de ces groupes contient exactement les énoncés du texte de procédure YBC 4663 vu précédemment, les énoncés étant cités dans les mêmes termes et dans le même ordre ${ }^{11}$. Le troisième de ces groupes correspond lui aussi à un texte de procédure connu, YBC 4662, qui se trouve également à l’Université Yale (voir Figure 1). Les autres groupes d'énoncés de notre catalogue devaient probablement être associés de la même manière à un texte des procédures. Le lien entre les catalogues et certains textes de procédure est donc très étroit. Mais dans quel sens ce lien a-t-il s'est-il établi ? Les énoncés des textes de procédures sont-ils extraits des catalogues, ou bien les catalogues sontils des compilations réalisées à partir de plusieurs textes de procédure ? La présence d'un colophon dans les catalogues, et l'organisation des énoncés en plusieurs groupes distincts conduit à privilégier la deuxième hypothèse ${ }^{12}$. Mais a question qui nous intéresse ici est autre, c'est celle de la fonction des catalogues. Les catalogues ont-ils été écrits dans le même but que les textes de procédure ? Les catalogues sont généralement considérés par les historiens modernes comme des répertoires d'exercices pédagogiques, tout comme les textes de procédure. Pourtant, le fait qu'ils soient structurés différemment incite à considérer la question de plus près. Rappelons tout d'abord que les catalogues ne donnent pas de consignes précises pour résoudre les problèmes. Par contre, leurs auteurs prenaient soin de noter le nombre exact de problèmes, et leur thématique. Par ailleurs, les catalogues sont des ensembles composites de plusieurs collections de problèmes provenant de textes de procédure différents, quoique portant sur le même thème. Plusieurs hypothèses sur la fonction des catalogues sont dès lors possibles. Une première hypothèse est suggérée par le lien étroit entre les catalogues et les textes de procédure, et par le fait que tous les catalogues connus sont de type S. Ces deux particularités évoquent les activités d'enseignement. Les catalogues pourraient résulter d'une volonté d'organiser le cursus mathématique et, peut-être, de le standardiser. Mais la présence des colophons, avec un compte des problèmes et une « étiquette » thématique, peut faire aussi penser à des pratiques d'archivage. En effet, au premier millénaire, soit dans des époques très postérieures à la période $\mathrm{pB}$, la pratique du colophon s'est considérablement développée en liaison avec la gestion des bibliothèques. On peut donc penser que les catalogues reflètent des pratiques de classification et d'archivage liées à l'apparition des premières bibliothèques ${ }^{13}$.

${ }^{9}$ Ici, « 31 sections » signifie « 31 énoncés » car chacun d’eux est écrit dans une section. Pour plus de détails concernant la relation entre sections et énoncés, qui n’est pas toujours aussi simple, voir (Proust en préparation).

${ }^{10}$ Pour la description précise de ces groupes et l'analyse de leur contenu mathématique, voir (Proust à paraître; Proust en préparation).

${ }^{11}$ Ce parallèle remarquable a été mis en évidence par O. Neugebauer et A. Sachs, qui ont publié les trois textes ensembles (Neugebauer and Sachs 1945, p. 66 ss.).

12 Pour plus de détails sur les arguments, voir (Proust en préparation).

${ }^{13}$ Cette idée est inspirée du travail de J.-J. Glassner sur les textes de divination (Glassner 2009). 
En toutes hypothèses, les catalogues résultent d'une démarche différente de celle des textes de procédure. Il s’agit non pas de demander au lecteur de résoudre des problèmes, mais en quelque sorte de décrire un état de l'art. Alors que les textes de procédure ont un caractère prescriptif, les catalogues apparaissent plutôt comme descriptifs. Du point de vue de leur statut pragmatique, ils se rapprochent des index, des inventaires, des tables des matières, ou encore des programmes d'enseignement officiels.

\section{Des listes pour explorer : les textes de séries}

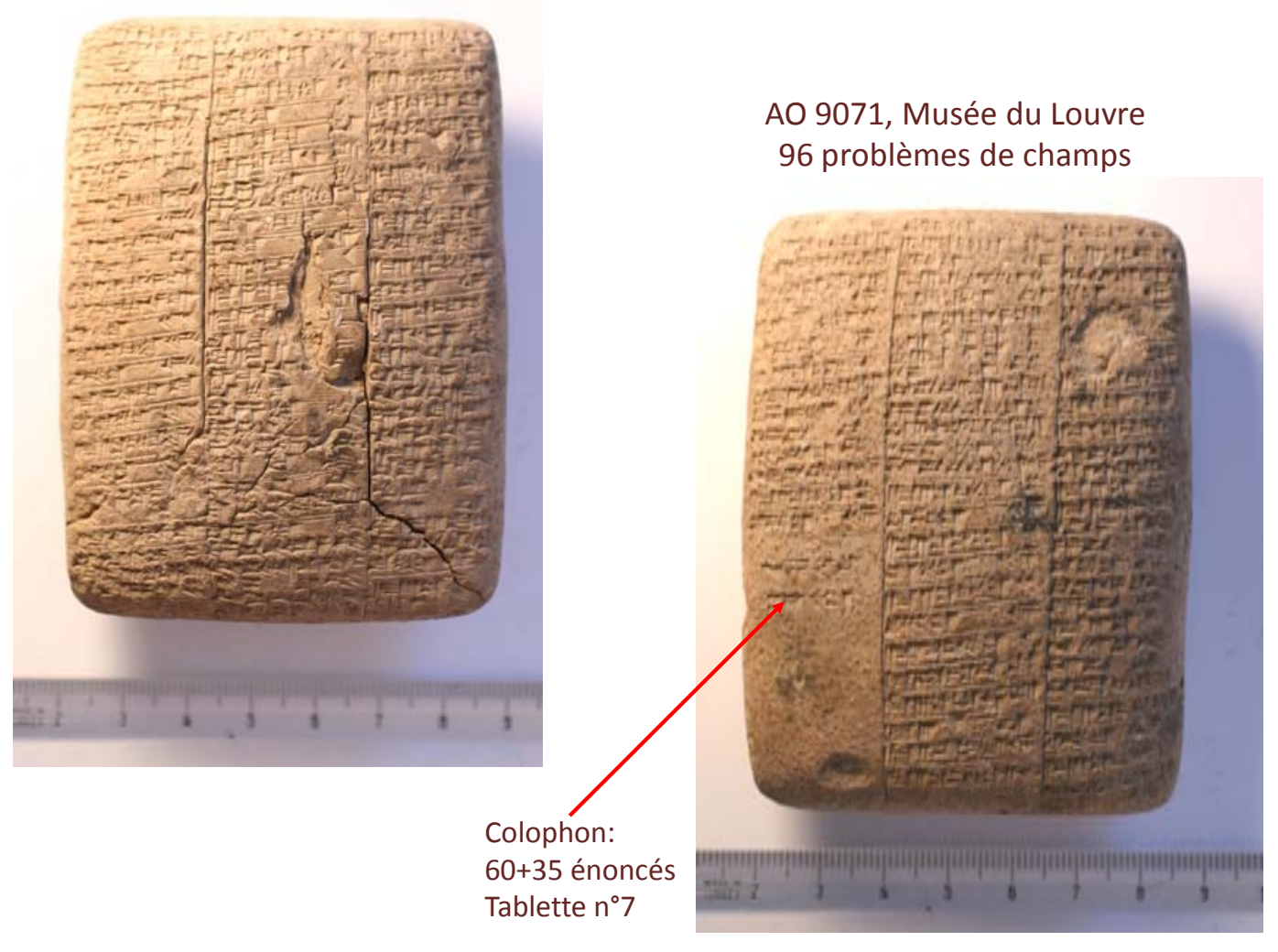

Figure 5 : tablette AO 9071, Musée du Louvre 
Souvent confondues avec les catalogues, les séries témoignent d'une toute autre démarche. Les séries mathématiques sont des listes d’énoncés de problèmes, tout comme les catalogues, mais ces listes sont beaucoup plus longues et couvrent plusieurs tablettes numérotées. La majorité des exemplaires se trouvent actuellement conservées dans la Yale Babylonian Collection, deux autres se trouvent à l'Oriental Institute de Chicago, deux autres au Vorderasiatisches Museum de Berlin ; tous ces textes ont été publiés par O. Neugebauer dans un chapitre de ses Mathematische Keilschrifttexte qui leur est consacré ${ }^{14}$. Le corpus s'est enrichi récemment de deux nouveaux exemplaires qui étaient restés ignorés dans les réserves du musée du Louvre ${ }^{15}$. Pour montrer les particularités des textes de séries, je vais me concentrer sur un des exemplaires du Louvre.

La tablette AO 9071 est une tablette multi-colonnes (type M). Elle se termine par un colophon qui donne le nombre de sections, comme dans les catalogues. Mais ici, ce nombre est plus important : 95. De plus, le colophon contient un numéro : tablette $n^{\circ} 7$.

Le début du texte montre comment se met en place le processus qui a permis de générer les centaines d'énoncés de la série de tablettes. Il est donc intéressant de l'examiner de près.

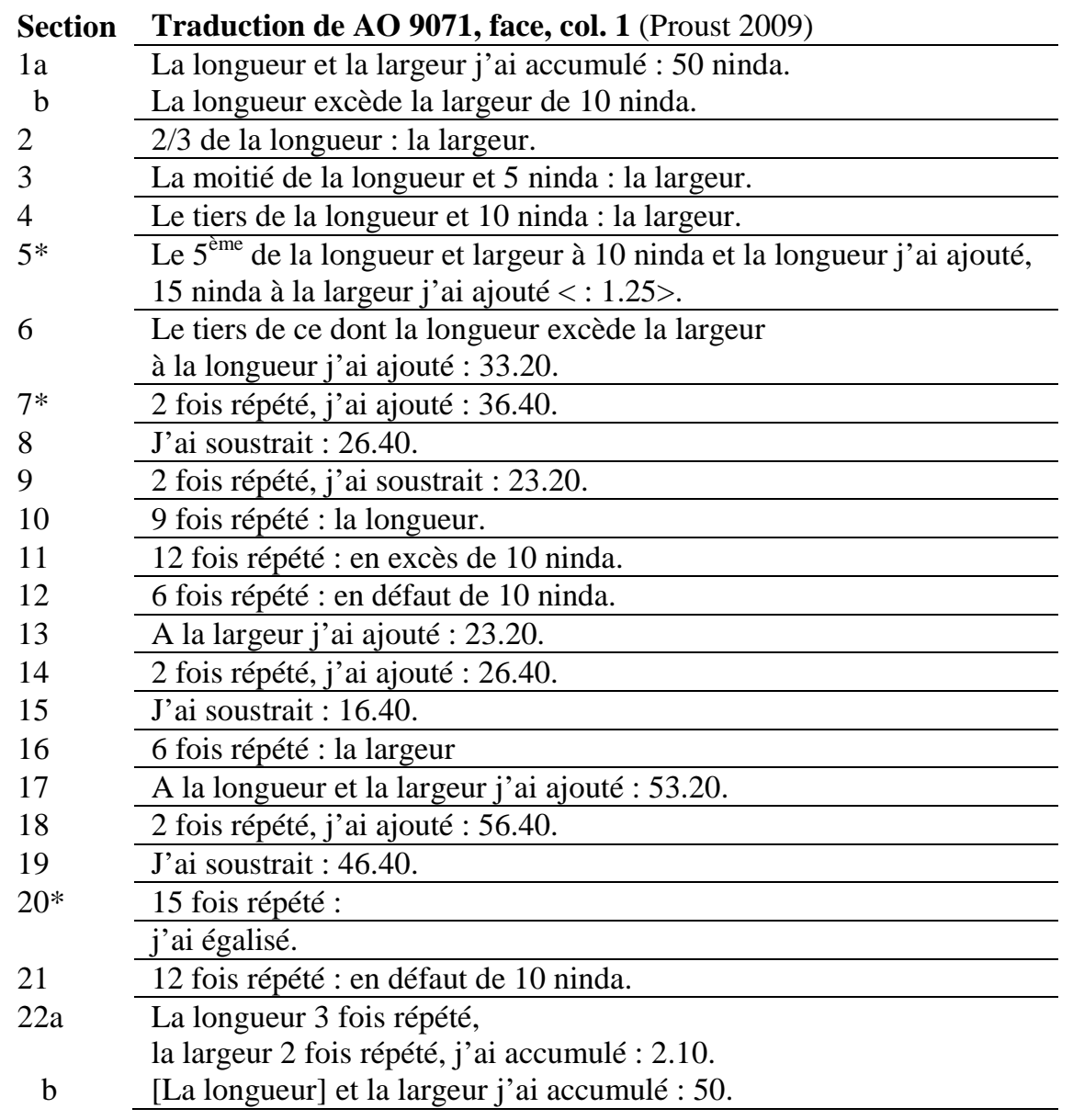

\footnotetext{
${ }^{14}$ Neugebauer 1935-7, ch. 7.

${ }^{15}$ Proust 2009.
} 
Le texte commence par un énoncé simple, commun dans les mathématiques pB. Cet énoncé donne deux relations linéaires entre la longueur et la largeur inconnues d’un rectangle, plus précisément leur somme et leur différence. En désignant par u la longueur (uš) et s la largeur (sag), on peut exprimer comme suit cet énoncé en langage moderne :

$$
\mathrm{u}+\mathrm{s}=50 \text { ninda et } \mathrm{u}-\mathrm{s}=10 \text { ninda. }
$$

La solution est toujours la même : la longueur est 30 ninda, la largeur est 20 ninda. On trouve cette même solution pour tous les problèmes de la tablette ainsi que de la série (ainsi, du reste, que d'une bonne partie des textes mathématiques cunéiformes).

Dès la deuxième section, on constate qu'une seule relation entre la longueur et la largeur est donnée : $2 / 3 \mathrm{u}=\mathrm{s}$. Une partie des informations manque donc. Il en est de même dans les sections 2 à 6 . En fait, l'information manquante est la première relation entre la longueur et la largeur, donnée ligne $1(\mathrm{u}+\mathrm{s}=50$ ninda que je désigne dans la suite, par commodité, équation $\mathrm{E}_{1}$ ).

L’énoncé 7 est encore plus lacunaire :

\#7 2 fois répété, j’ai ajouté : 36.40.

On ne sait pas ce qui est répété, ni à quoi on ajoute. Pour le savoir, comme précédemment, il faut rechercher des informations déjà données dans la section précédente :

\#6 Le tiers de ce dont la longueur excède la largeur à la longueur j'ai ajouté : 33.20 .

Dans le texte cunéiforme, aucun indice matériel ou typographique ne permet de déceler quels sont les éléments de la section 6 qui sont réutilisés dans la section 7. Cependant, l’habitude de ce genre de texte donne la clé assez facilement ${ }^{16}$. Ce qui est répété est « Le tiers de ce dont la longueur excède la largeur », soit une expression que je désigne par $\mathrm{P}$ (expression principale) dans ce qui suit. Ce à quoi on ajoute est «la longueur », une expression que je désigne par $\mathrm{S}$ (expression secondaire) dans ce qui suit. La première relation entre la longueur et la largeur est toujours la même, à savoir la relation $\mathrm{E}_{1}$ donnée dans la section 1.

Les énoncés 8 à 12 sont construits de la même façon. Ils donnent des relations entre les expressions $\mathrm{P}$ et $\mathrm{S}$, mais sans expliciter ni $\mathrm{P}$, ni $\mathrm{S}$, ni l'équation $\mathrm{E}_{1}$. Ainsi, les sections 7 à 12 dépendent de la section 6 pour la définition de $\mathrm{P}$ et de $\mathrm{S}$, et de la section 1 pour la définition de $E_{1}$.

Dans la section 13, la valeur de ce à quoi on ajoute (expression secondaire S) est changée : c'est maintenant «la largeur ». Les sections 14-15-16 donnent d'autres relations entre P et cette nouvelle valeur de S. Ainsi, les sections 13 à 16 dépendent des sections 13, 6 et 1. Dans la section 17, S change encore, et les sections 18 à 21 utilisent cette nouvelle valeur de $\mathrm{S}$.

\footnotetext{
${ }^{16}$ Neugebauer a décrit les étapes de l'enquête quasi policière qui l’a amené à percer le secret des textes de série, c'est-à-dire à comprendre les principes de génération des énoncés, dans un article passionnant écrit dès 1934 (Neugebauer 1934-6). Voir aussi (Neugebauer 1951 § 33, p. 63-66).
} 
Dans la section 22, la valeur de $\mathrm{P}$ (et de $\mathrm{E}_{1}$ ) est changée, et le même processus est itéré : variations de $\mathrm{S}$, puis, pour chaque valeur de $\mathrm{S}$, variations de la relation entre $\mathrm{P}$ et $\mathrm{S}$.

Dans la section 35, la valeur de $\mathrm{E}_{1}$ est encore changée, et le même processus s'enclenche à nouveau : variations de $\mathrm{P}$, puis, pour chaque valeur de $\mathrm{P}$, variations de $\mathrm{S}$, puis, pour chaque valeur de $\mathrm{S}$, variations de la relation entre $\mathrm{P}$ et $\mathrm{S}$.

Les valeurs de $\mathrm{E}_{1}$ et de $\mathrm{P}$ sont de plus en plus compliquées, les énoncés de plus en plus lacunaires. Ainsi, plus on avance dans le texte, plus les énoncé écrits sont brefs et les énoncés représentés compliqués. Par exemple, la section 59 est réduite à quatre signes (ba-zi-ma 45), qui signifient :

J'ai soustrait: 45

Une fois que toute l'information à laquelle renvoie cette formule des plus laconiques a été récupérée dans les différents points du texte où elle se trouve, on obtient un énoncé dont la représentation selon le formalisme moderne est la suivante :

$\left\{\begin{array}{l}(3 x+2 y) \frac{1}{13}+x=40 \\ -\left\{\left\{\left\{[(x+25)+(y+1.30-x)+(x+y+35)] \frac{1}{11}+4 x\right\} \frac{1}{7}+y\right\rangle \times 2 \times \frac{1}{16}-(x+2 y)+x+y+(3 x-2 y)\right\} \frac{1}{7}+(x+y)=45\end{array}\right.$

On obtient ainsi une structure arborescente à 4 niveaux. Dans chacun de ces niveaux sont définis des segments de l'énoncé. Ces niveaux sont, en allant du tronc vers les extrémités de l'arborescence :

Niveau 4 : définition de l'équation $\mathrm{E}_{1}$

Niveau 3 : définition de l'expression principale $\mathrm{P}$

Niveau 2 : définition de l'expression secondaire $S$

Niveau 1 : définition de la relation entre $P$ et $S$

Chaque segment subit des variations tour à tour.

L'équation $E_{1}$ subit plusieurs variations. A l'échelle de la tablette AO 9071, on trouve 5 variantes (d'autres variantes interviennent à l'échelle de la série) : 


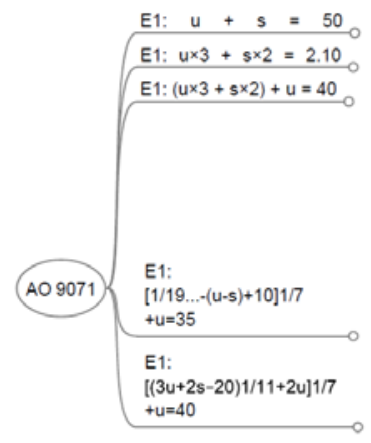

Figure 6 : variations de niveau 4

Pour chaque variante l'équation $\mathrm{E}_{1}$, l'expression principale $\mathrm{P}$ subit plusieurs variations (entre 1 et 4$)$ :

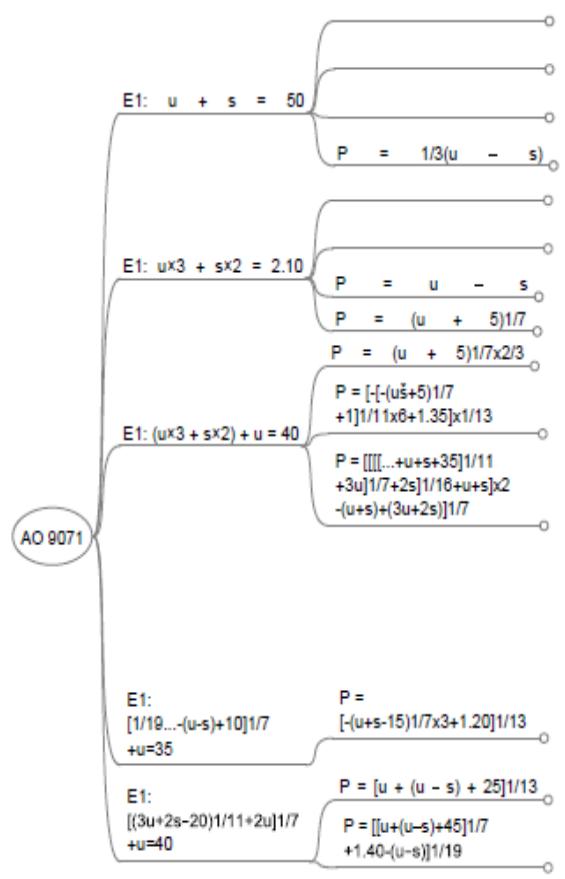

Figure 7 : variations de niveau 3 
Pour chaque variante de l'expression principale $\mathrm{P}$, l'expression secondaire $\mathrm{S}$ subit plusieurs variations (entre 1 et 4 variantes assez régulières) :

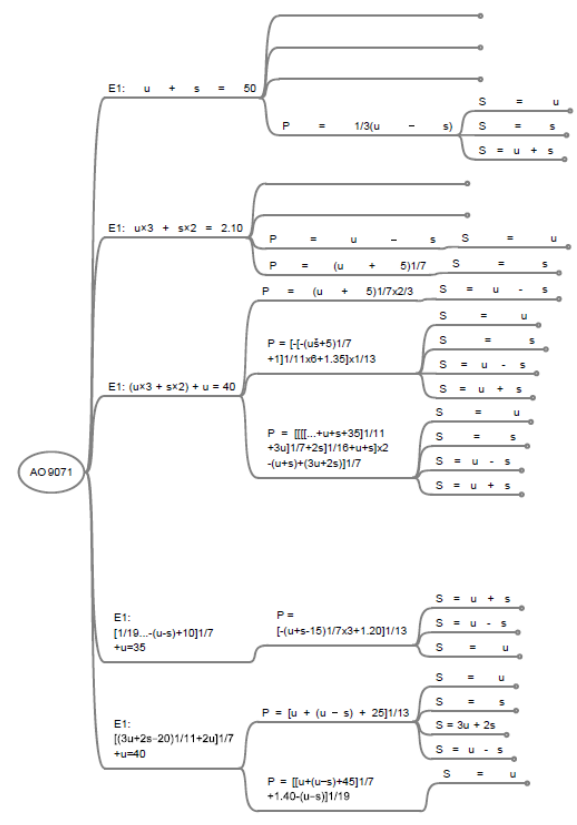

Figure 7 : variations de niveau 2 
Pour chaque variante l'expression secondaire $S$, la relation entre $\mathrm{S}$ et $\mathrm{P}$ subit plusieurs variations, elles aussi assez régulières (entre 1 et 6 ) :

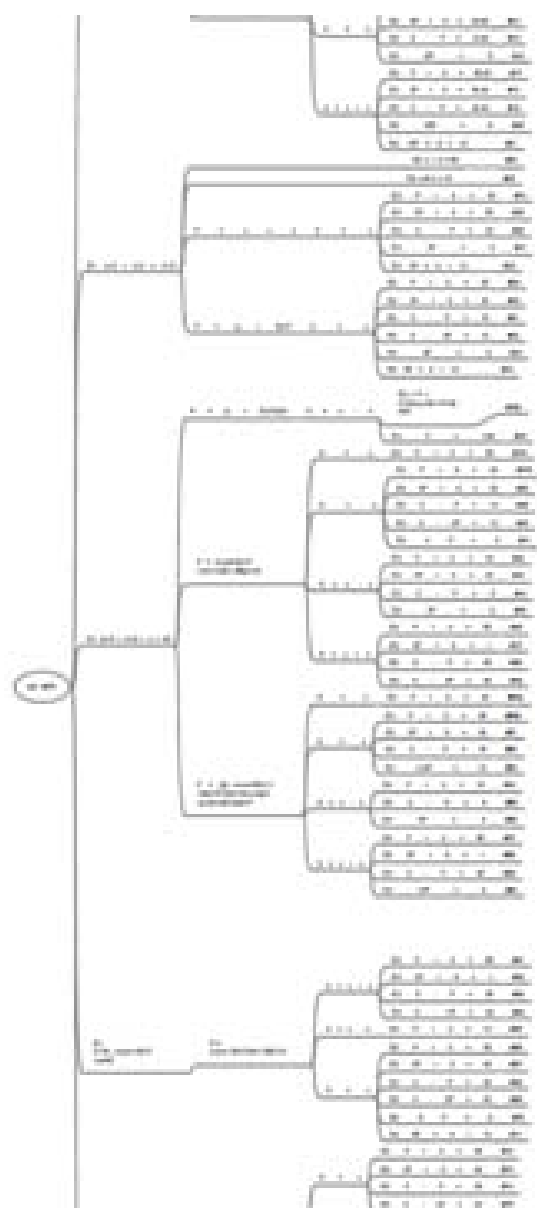

Figure 8 : variations de niveau 1

Sur cette structure arborescente, se greffe une information dont la répartition répond au principe d'économie qu'on a vu fonctionner ci-dessus dans le début du texte: toute information donnée à un niveau de l'arborescence est omise dans les niveaux qui en dépendent. En conséquence, lorsqu'on lit l'énoncé écrit dans une section, il faut remonter dans le texte pour chercher l'information donnée dans les niveaux parents. Dans le cas de la section 59, l'information se trouve en quatre points différents. Circuler dans le texte pour récupérer l'information utile n'était sans doute pas un exercice facile à la portée d'un scribe ordinaire. Les lecteurs ou usager des séries devaient baigner une culture du texte très particulière.

La combinaison d'une structure de liste arborescente avec une répartition rationalisée de l'information donne des potentialités à l'écriture mathématique très supérieure à celle que l'on trouve dans les autres textes mathématiques. Elle permet une compression considérable de l'information. Elle permet aussi l'expression d'opérations hiérarchisées sur plusieurs niveaux (jusqu'à 5 dans le \#59 de la tablette du Louvre) que nous représentons aujourd'hui par des niveaux de parenthèse. 
D’un point de vue mathématique, le procédé de génération des énoncés revient à remplacer certaines combinaisons des inconnues par d'autres combinaisons de même nature (ici, ces combinaisons sont linéaires, mais dans d'autres textes, elles peuvent être quadratiques). Ces substitutions systématiques permettent de produire à l'infini de nouveaux énoncés mathématiquement équivalents (mêmes inconnues, même degré, mêmes solutions). Soulignons que si le procédé permet de contrôler la fabrication des énoncés, il ne permet pas de contrôler les procédures de résolution. En effet, la liste n’est pas organisée en fonction des méthodes de résolution, comme dans les textes de procédure et les catalogues, mais en fonction des caractéristiques formelles des énoncés. Et de fait, si les énoncés initiaux dans chaque tablette semblent relativement communs, on obtient au fil des listes des énoncés de plus en plus complexes, voire extravagants. Pour certains de ces problèmes, on peut douter sérieusement que les scribes aient été en mesure des les résoudre ${ }^{17}$.

Nous avons donc devant les yeux un exemple de liste qui semble poursuivre d'autres buts que ceux qui ont été repérés dans les cas précédents : ni prescriptifs, ni descriptifs, ils relèvent d'un autre statut pragmatique. Qu'était-il donc attendu du lecteur de ces gigantesques listes ? Ce n'est pas la résolution des problèmes, puisque celle-ci est manifestement impossible dans de nombreux cas. Ce n'est pas l'archivage du matériel existant, puisque la plupart des problèmes ne sont pas attestés ailleurs. Les séries se présentent plutôt comme un outil heuristique dont le but est d'explorer les propriétés d'un objet mathématique, l'énoncé de problème.

A qui s'adressaient les séries ? Il me paraît clair que les séries ne sont pas des collections de problèmes destinés à l'éducation mathématique des étudiants d'écoles de scribes. Les séries ont clairement été écrites par des érudits d'un très haut niveau, connaissant parfaitement la tradition mathématique de leur époque, et maîtrisant avec virtuosité l'écriture cunéiforme. Les destinataires n’étaient sans doute pas leurs étudiants, mais leurs pairs.

\section{Diverses raisons d'écrire des listes}

Voir les listes de problèmes comme des manuels d'exercices pour l'enseignement est non seulement réducteur, comme indiqué en introduction, mais surtout conduit à privilégier certaines interprétations et à en occulter d'autres. Or les exemples qui ont été présentés ici montrent que le réseau de communication dans lequel s'inscrit un texte mathématique est beaucoup plus riche et complexe que la simple transmission de maître à élève. Quand on regarde les textes mathématiques en tant que listes, on constate qu'ils ne répondent pas tous aux mêmes "projets d'écriture », c'est-à-dire qu'ils ne s’adressent pas aux mêmes types de lecteurs, et qu'ils ne poursuivent pas les mêmes objectifs. Certains s'apparentent à des manuels, d'autres à des inventaires, d'autres encore ressemblent plutôt à des entreprises exploratoires.

${ }^{17}$ Le problème 59 évoqué ci-dessus en est un exemple. On trouve même des problèmes de degrés 4 ou 5 non réductibles à des degrés plus bas dans d'autres les textes de séries. 
Ces questions pourraient être importantes du point de vue de l'histoire intellectuelle de la Mésopotamie à la fin de la période paléo-babylonienne. En effet, les séries semblent ne pas avoir été élaborées dans le même contexte que les catalogues et les textes de procédure. Les indices paléographiques plaident pour une datation relativement tardive des séries, c'est-à-dire de la fin de l'époque paléo-babylonienne. D’autres indices, tels que l'organisation en séries numérotées, l'usage d'une langue complètement artificielle imitant le sumérien, suggère que ces textes pourraient venir de la région centrale de la Mésopotamie, par exemple Kiš ou Sippar. En même temps, leurs auteurs connaissaient parfaitement la tradition mathématique du Sud, dont ils reproduisaient la terminologie et les thématiques. Les séries ont sans doute été écrites alors que la tradition mathématique s'était déjà pleinement développée. Elles témoignent en tout cas d'une connaissance profonde de la tradition mathématique pB. En même temps elles sont, par certains aspects, totalement innovantes.

Biblio

Bernard, Alain and Christine Proust. in progress. "Studying ancient scientific sources produced in an educational context: problems and perspectives." Boston Studies.

Delnero, Paul. 2010. "Sumerian Literary Catalogues and the Scribal Curriculum." Zeitschrift für Assyriologie und Vorderasiatische Archäologie 100:32-55.

Glassner, Jean-Jacques. 2009. "Ecrire des livres à l'époque paléo-babylonienne: le traité d'extispicine." Zeitschrift für Assyriologie und Vorderasiatische Archäologie 99:1-81.

Goetze, Albrecht. 1945. "The Akkadian dialects of the Old-Babylonian mathematical texts." Pp. 146-151 (ch. IV) in Mathematical Cuneiform Texts, vol. 29, American Oriental Society, edited by O. Neugebauer and A. J. Sachs. New Haven: American Oriental Series \& American Schools of Oriental Research.

Goody, Jack. 1977. The Domestication of the Savage Mind. Cambridge-London-New York Cambridge university Press.

Høyrup, Jens. 2002. Lengths, Widths, Surfaces. A Portrait of Old Babylonian Algebra and its Kin. Berlin \& Londres: Springer.

Luc, Christophe, Mustapha Mojahid, Marie-Paule Pery-Woodley, and Jacques Virbel. 2000. "Les énumérations : structures visuelles, syntaxiques et rhétoriques." Pp. 21-40 in III Colloque International sur le Document Electronique CIDE 2000, edited by M. Gaio and E. Trupin. Lyon: Europia Productions.

Neugebauer, Otto. 1934-6. "Serientexte in der babylonischen Mathematik." Quellen und Studien zur Geschichte der Mathematik, Astronomie und Physik B 3:106-114.

—. 1935-7. Mathematische Keilschrifttexte I-III. Berlin: Springer.

-. 1951. The exact sciences in antiquity. London: Oxford University Press.

Neugebauer, Otto and Abraham J. Sachs. 1945. Mathematical Cuneiform Texts, vol. 29. New Haven: American Oriental Series \& American Schools of Oriental Research

Proust, Christine. 2009. "Deux nouvelles tablettes mathématiques du Louvre : AO 9071 et AO 9072." Zeitschrift für Assyriologie und Vorderasiatische Archäologie 99:167-232.

—. 2012. "Reading colophons from Mesopotamian clay-tablets dealing with mathematics." Pp. *** in Questions linked to text, vol. ***, Natur, Technik, Medizin (NTM), edited by K. Chemla: Natur, Technik, Medizin (NTM). 
—. à paraître. "Reading incipit and colophons from Mesopotamian clay-tablets dealing with mathematics." in Questions linked to text, Natur, Technik, Medizin (NTM), edited by K. Chemla: Natur, Technik, Medizin (NTM).

- en préparation. L'art des listes dans les mathématiques cunéiformes. Approche linguistique, épistémologique et historiographique des listes d'équations (Mésopotamie, début du deuxième millénaire avant notre ère).

Sève, Bernard. 2010. De haut en bas. Philosophie des listes. Paris Editions du Seuil, coll. "L'ordre philosophique".

Tinney, Steve. 1999. "On the curricular setting of Sumerian literature." Iraq 61:159-172.

Virbel, Jacques. 1997. "Aspects du contrôle des structures textuelles." Pp. 251-272 in Perception auditive et compréhension du langage, edited by J. Lambert and J.-L. Nespoulous: Solal.

—. à paraître. "Les énumérations textuelles (preprint en français)." in Introduction to textology via scientific writings, edited by K. Chemla and J. Virbel. 